\title{
Cilioretinal collateral circulation after occlusion of the central retinal artery
}

\author{
MICHAEL F MARMOR,' LEE M JAMPOL, ${ }^{2}$ AND LISA WOHL \\ From the 'Division of Ophthalmology, Stanford University, and the Ophthalmology Section, Veterans \\ Administration Medical Center, Palo Alto, CA, and the ${ }^{2}$ Department of Ophthalmology, \\ Northwestern University Medical School, Chicago, IL, USA
}

SUMmary Two elderly patients were noted to have unilateral central retinal arterial narrowing with cilioretinal arterial collaterals supplying the bulk of the flow to the inner retina. One patient had excellent acuity and normal pupillary reactions; the other had marked impairment of vision and an afferent pupillary defect. Both patients were asymptomatic. We suggest that these patients had gradual or partial central retinal arterial occlusion which allowed the development of a collateral arterial supply from the ciliary system.

Occlusion of the central retinal artery is most often an obvious event in which vision is abruptly lost. If the occlusion is only partial or brief, significant recovery of vision may occur. More often, however, permanent retinal damage results, and the eye remains amaurotic regardless of whether the vessels reperfuse. Retinal or anterior segment neovascularisation develops rarely, presumably because infarcted tissue does not stimulate new vessel growth. The occasional development of arterial collaterals after arterial occlusion has been recognised for many years, ${ }^{1-4}$ most often after embolic branch artery occlusion. We report two unusual cases in which unrecognised, and presumably gradual, blockage of the central retinal artery led to the development of a nearly complete cilioretinal collateral circulation.

\section{Case reports}

CASE 1

A 69-year-old white male complained of gradual painless visual loss on the right. He denied glaucoma, amblyopia, or other previous eye disease. In 1975 he had both a coronary artery bypass graft and a left carotid endarterectomy, but he has had no strokes or myocardial infarctions and did not have hypertension. His best corrected visual acuity was $20 / 200$ OD, 20/20 OS, and visual field testing showed no scotomas. The pupils reacted normally and equally.

Correspondence to Michael F Marmor, MD, Ophthalmology Section (112B1), Veterans Administration Medical Center, 3801 Miranda Avenue, Palo Alto, CA 94304, USA.
Applanation tensions were $15 \mathrm{mmHg}$ OD, $18 \mathrm{mmHg}$ OS. Both lenses had early nuclear sclerosis, with a prominent posterior subcapsular plaque present on the right accounting for the decreased vision. After extracapsular cataract extraction and lens implantation on the right, his corrected acuity was $20 / 20$ in both eyes.

Both fundi (Figs. 1A, B) showed tigroid pigmentation and normal maculae. The discs were flat and pink; a superficial hazy whitish lesion was noted just superior to the right disc. The veins were of normal caliber. On the left the arteries were mildly narrowed everywhere except over the disc, where they were markedly constricted. Prominent collaterals arose from the edge of the disc to join the arterial trunks beyond the disc margin. Fluorescein angiography (Figs. 2A, B, C) showed that the superior nasal retinal artery filled directly, but all other arterial branches received their blood supply from the ciliary vessels. No leakage or neovascularisation was seen in the late views. On the right the temporal arteries were mildly narrowed, while the nasal ones showed severe constriction. An embolus or plaque was visible at a trifurcation of the superior nasal artery near the disc margin. Fluorescein angiography (Figs. $3 \mathrm{~A}, \mathrm{~B}$ ) revealed that collaterals, unseen in the fundus photographs, were developing to the middle branch of this trifurcation.

CASE 2

A 67-year-old black female requested new glasses but had no specific visual complaints. Medical history 


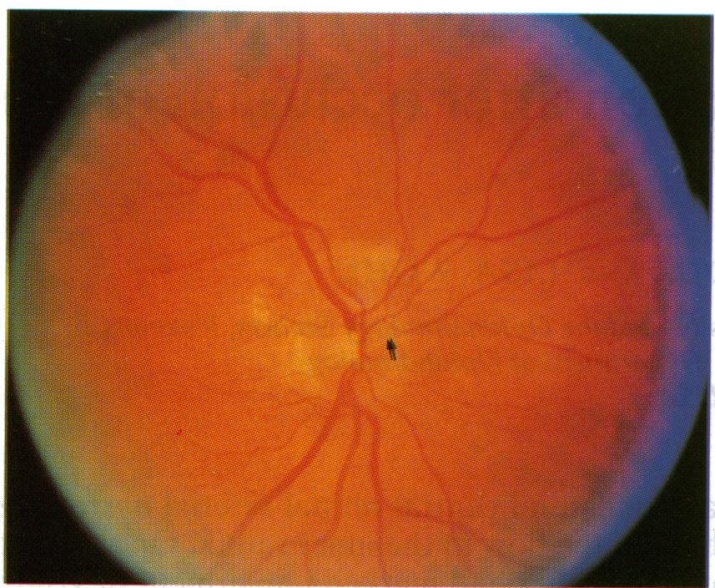

Fig. 1A

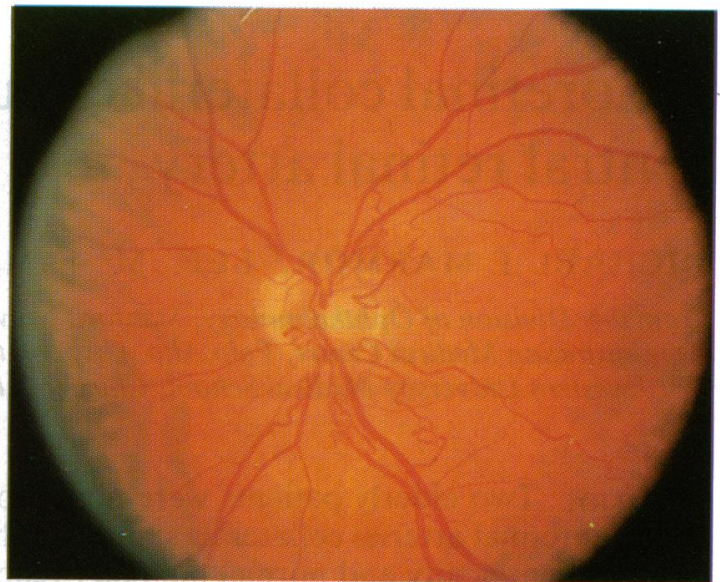

Fig. 1B

Fig 1 Fundi of case I. A. Right eye. The arteries are narrowed slightly on the temporal side and markedly on the nasal side. Arrow points to a plaque or embolus at an arterial trifurcation. B. Left eye. Tortuous cilioretinal collaterals supply the major arterioles.

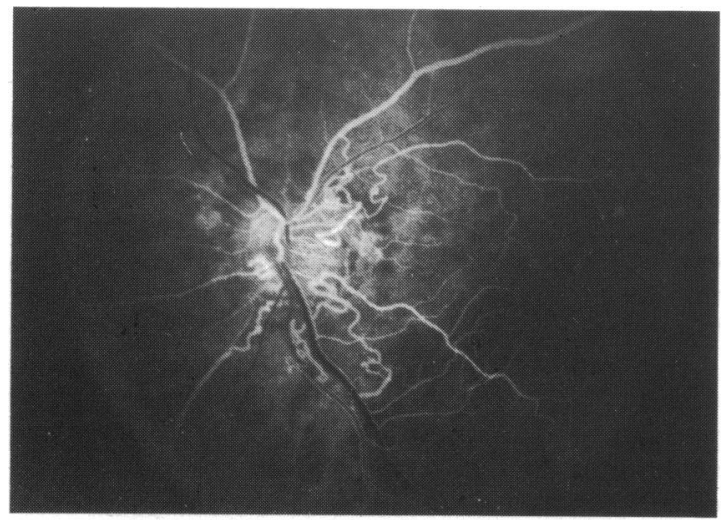

Fig. 2A

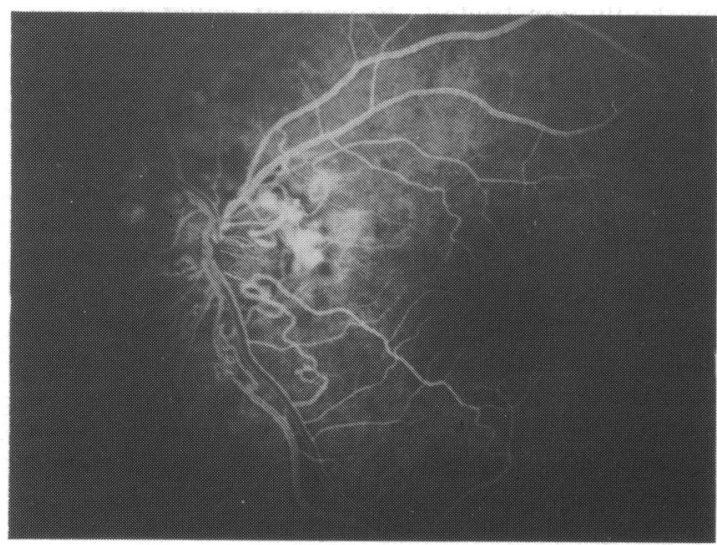

Fig. 2B revealed moderate hypertension with poor medical compliance. $X$-rays of the orbit, optic foramen, and skull were normal. Her best corrected visual-acuity was $20 / 40 \mathrm{OD}, 20 / 200 \mathrm{OS}$, and there was a positive afferent pupillary defect on the left. Examination of the anterior segment was otherwise unremarkable, and applanation tensions were $26 \mathrm{mmHg}$ OD, 28 mmHg OS. Goldmann perimetry showed the visual fields to be full on the right but constricted with a loss of nasal field on the left.

Both fundi (Figs. 4A, B) showed normal peripheral pigmentation and macular architecture. The disc appeared normal on the right but showed pallor on the left. The vascular calibre was normal on the right, though some mild arteriosclerosis was present. On

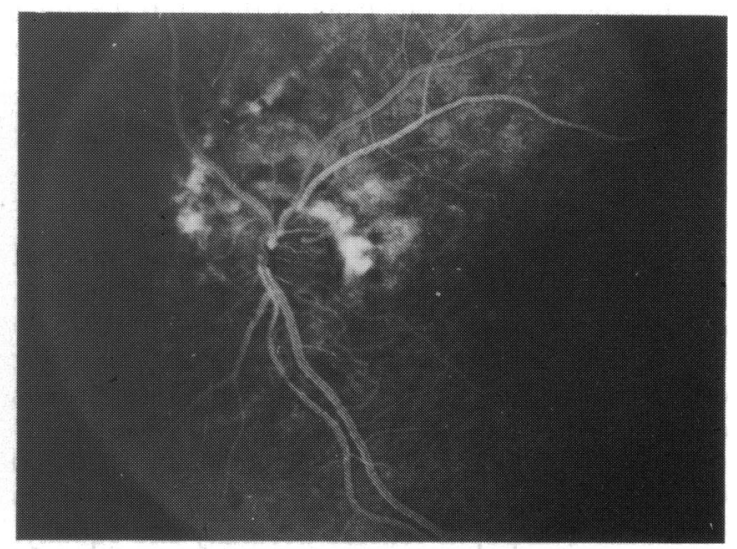

Fig. 2C

Fig. 2 Fluorescein angiograms of the left eye of case 1. All the arterioles, except the superior nasal one, fill from the cilioretinal circulation. A. Arterial phase. B. Arteriovenous phase. C. Venous phase. 


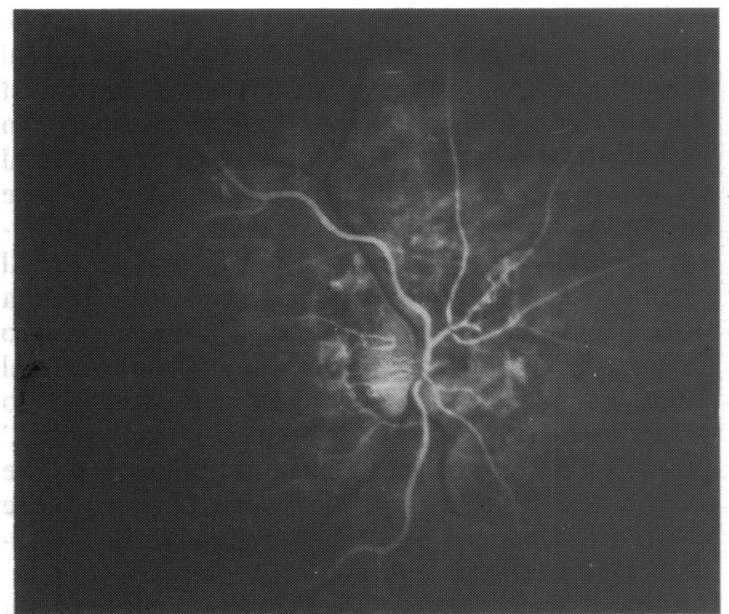

Fig. 3A

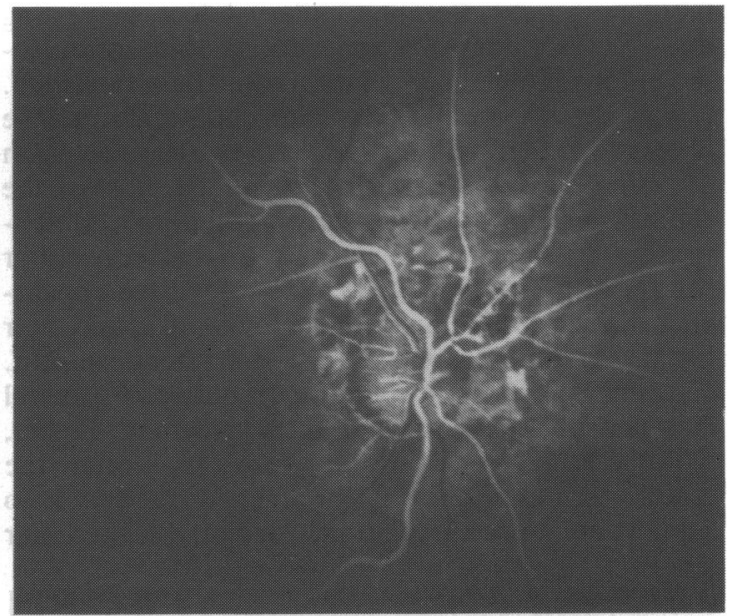

Fig. 3B

Fig. 3 Fluorescein angiograms of the right eye of case 1. Early cilioretinal collaterals join a branch arteriole beyond the obstruction identified in Fig. 1A. A. Early arterial phase. B. Late arterial phase.

the left the veins showed normal calibre, but the arteries were moderately narrowed. Over the disc the branch arteries from the central retinal artery became mere threads and were joined by collaterals arising from the edge of the disc. Fluorescein angiography of the left eye (Figs. 5A, B, C) showed that there was a small degree of filling from the central retinal artery, but the arteriolar collaterals supplied much of the arterial flow. There was no neovascularisation or leakage of fluorescein.

\section{Discussion}

These cases are unusual in that nearly complete

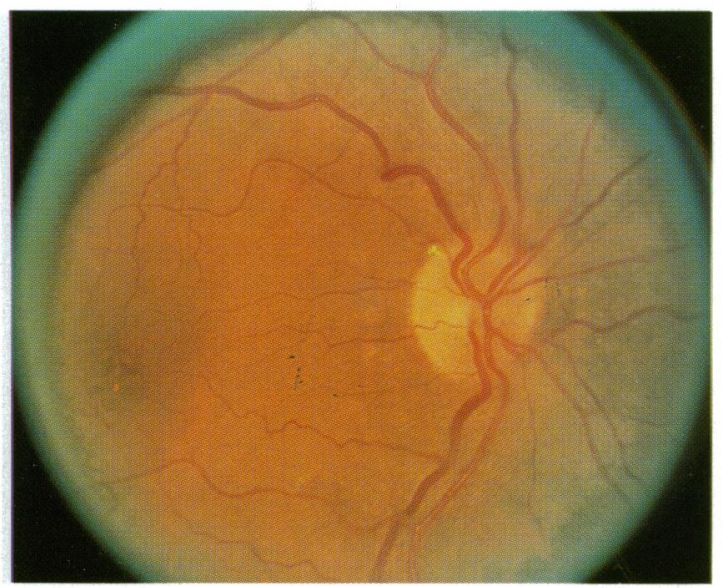

Fig. 4A occlusion of the central retinal artery occurred without severe loss of vision. Either the obstruction must have developed gradually, so that there was no episode of sudden visual loss, or the initial episode of obstruction was overlooked and the vessels opened sufficiently to allow partial recovery. As the central retinal artery narrowed, or after its occlusion, fine capillary connections between the ciliary and retinal circulations expanded to augment the blood flow to the retina. Remarkably, the visual acuity of the affected eye in case 1 remained 20/20. This was in fact the patient's good eye; his presenting complaint was poor vision from the cataract on the right. The visual acuity of the affected eye was $20 / 200$ in case 2 , and

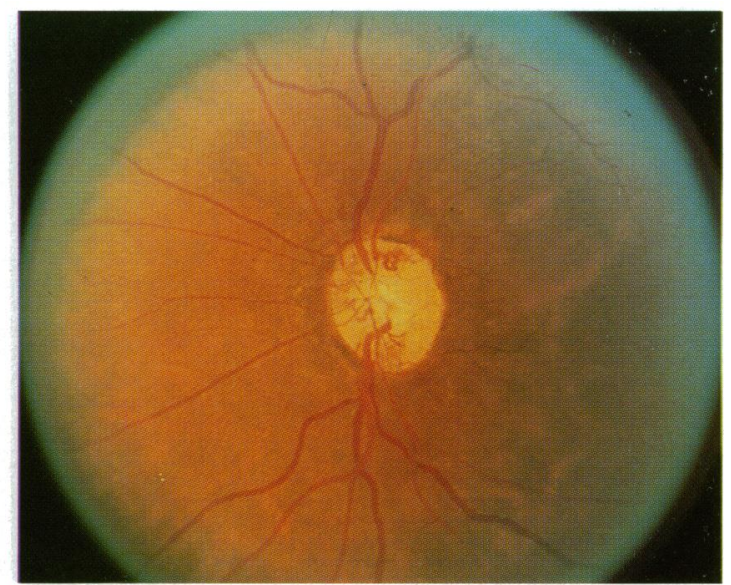

Fig. 4B

Fig. 4 Fundi of case 2. A. Right eye. B. Left eye. There is marked narrowing at the origin of the retinal arteries, and cilioretinal collaterals bypass the constriction. 
there was a mild afferent pupillary defect, suggesting that a greater degree of inner retinal damage occurred before sufficient circulation could be established.

The development of arterial collaterals can be observed experimentally within days after occlusion of retinal vessels with emboli. ${ }^{2}$ Indeed most of the clinical descriptions have shown collaterals circumventing a visible blockage of a retinal artery, either central or peripheral. Jensen' described the appearance of such collaterals within a few weeks, and their formation probably begins very soon after occlusion. Why are they not seen more frequently? Several factors probably contribute to their scarcity. Firstly, if the vascular occlusion is severe (as is often the case), vascular tissue will be damaged and unable to support collateral circulation. Secondly, if the inner

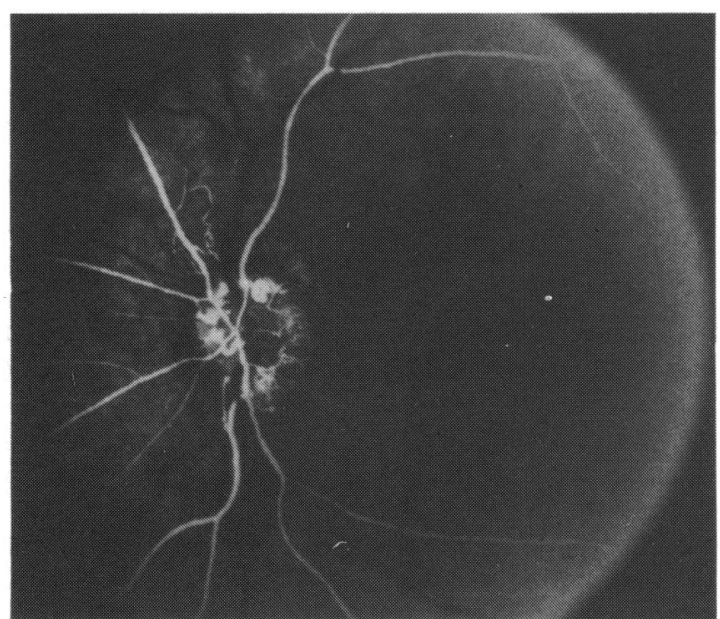

Fig. 5A

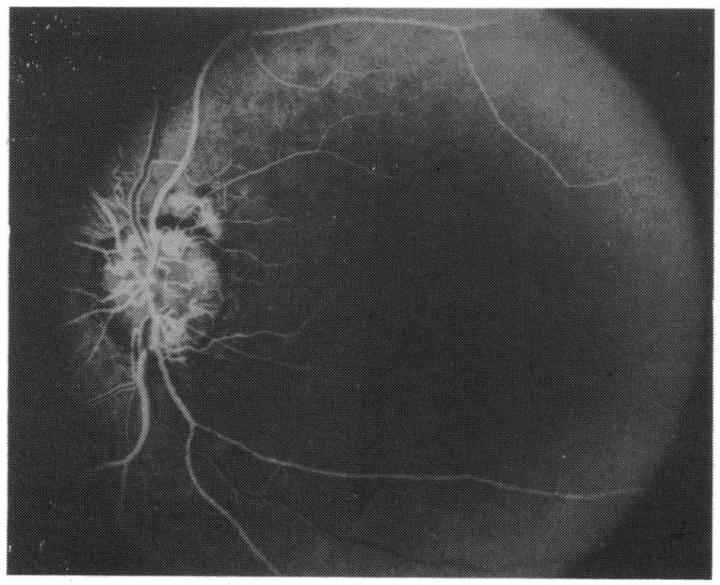

Fig. 5B retinal neurons are severely damaged, metabolic demand may be insufficient to justify collateral formation even if the vasculature survives. Thirdly, if the blockade is relieved (for example by passage of an embolism or relief of spasm) the need for collateral circulations will be eliminated. Fourthly, if the pathophysiology of occlusion included more proximal vascular disease, such as occlusion of the internal carotid artery, there might be concomitant ischaemia of the choroidal circulation and insufficient flow to support collaterals. For example, chronic retinal ischaemia from carotid occlusive disease may lead to a fundus picture of venous congestion and stasis, possibly on the basis of ischaemic damage to the vasculature. Since the site of occlusion is outside the orbit, the ischaemia is not correctable by the formation of cilioretinal collaterals.

Only if these various conditions are absent, if a relatively focal blockage exists in the retinal arterial tree, and if sufficient retinal and choroidal flow exists to maintain at least minimal retinal and vascular viability, will collaterals be able to form. This set of conditions could exist after an embolic block which still allows a trickle of blood to get through. Indeed this seems to be the situation in the right eye of case 1 , in which a branch arteriole, partly occluded by an embolus, is just beginning to develop collaterals. Alternatively, gradual closure of the retinal vessel by sclerosis or thrombus formation, or by recurrent tiny emboli, might encourage the widening of collateral channels. Case 1 shows clearly that once collaterals have developed, good retinal function can be maintained through a ciliary supply. The existence of occasional patients with absent retinal arteries and a congenital cilioretinal arterial supply to the retina

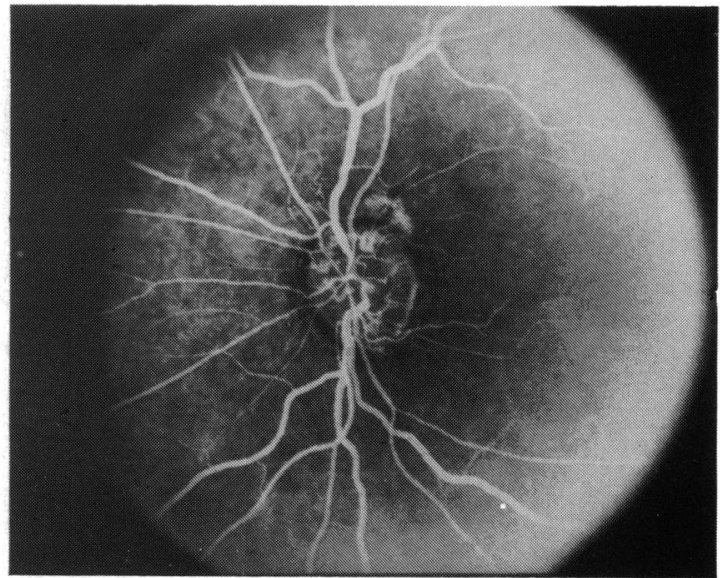

Fig. 5C

Fig. 5 Fluorescein angiograms of the left eye of case 2. The presence of cilioretinal arteriolar collaterals is confirmed. A. Arterial phase. B. Arteriovenous phase. C. Venous phase. 
confirms this observation. We consider the lesions in our patients were clearly acquired and not congenital.

In cases where there is no history of sudden visual loss, arterial collaterals must be distinguished from other vascular abnormalities. Venous collaterals may develop in conditions such as central retinal vein occlusion or an optic nerve sheath mengingoma which blocks venous drainage, ${ }^{\circ}$ but the venous nature of the collateral circulation should be evident on fluorescein angiography. Arterial collaterals fill immediately as fluorescein enters the eye and before the venous phase has begun, whereas venous collaterals will be seen to fill during the venous phases of the angiogram. Many vascular anomalies of the retina and optic disc have been described, such as familial arteriolar tortuosity ${ }^{7}$ or prepapillary loops. ${ }^{8}$ These are unlikely to be confused with the characteristic pattern formed by tortuous collaterals arching over a zone of narrowed or absent retinal artery. Collateral vessels may resemble neovascularisation, and this distinction is important because the presence of neovascular growth would signify continued ischaemia and a possible need for photocoagulation therapy. Furthermore both may be present concomitantly." Retinal and optic disc neovascularisation is rather rare after retinal artery occlusion and more often seen in the presence of carotid occlusive disease. ${ }^{111}$ However, since carotid disease is not uncommon in patients with retinal artery occlusive disease, separating the contribution from each entity may not always be easy. New vessels, like collaterals, do not ordinarily develop after a complete retinal artery occlusion, because little viable retinal tissue remains. If the occlusion is more gradual, or conditioned by preexisting central ischaemia, then neovascularisation of the retina and anterior segment may develop. The diagnosis can be confirmed by fluorescein angiography, which will show a leakage of dye from neovascularisation but not from arterial collaterals (as in these present cases), which retain tight junctions.

Gary Lissner, MD, allowed us to report case 2.

\section{References}

1 Jensen VA. Anastomosis formation after embolism of the central retinal artery. Acta Ophthalmol (Kbh) 1938; 16: 485-502.

2 Henkind P. Ballotini occlusion of retinal arteries. Collateral vessels. BrJ Ophthalmol 1966; 50: 482-95.

3 Karjalainen $\mathrm{K}$. Occlusion of the central retinal artery and retinal branch arterioles. Acta Ophthalmol (Kbh) 1971; 109 (suppl): 9-66.

4 Arruga J, Sanders MD. Ophthalmologic findings in 70 patients with evidence of. retinal embolism. Ophthalmol (Rochester) 1982; 89: 1336-47.

5 Kearns TP, Hollenhorst RW. Venous stasis retinopathy of occlusive disease of the carotid artery. Mayo Clin Proc 1963; 38: 304-12.

6 Hollenhorst RW Jr, Hollenhorst RW Sr, MacCarty CS. Visual prognosis of optic nerve sheath meningiomas producing shunt vessels on the optic disk: the Hoyt-Spencer syndrome. Trans Am Ophthalmol Soc 1977; 75: 141-61.

7 Goldberg MF, Pollack IP, Green WR. Familial retinal arteriolar tortuosity with retinal hemorrhage. Am J Ophthalmol 1972; 73: 183-91.

8 Degenhart W, Brown GC, Augsburger JJ, Magargal L. Prepapillary vascular loops. Ophthalmology (Rochester) 1981; 88: $1126-31$.

9 Willerson D Jr, Aaberg, TM. Acute central retinal artery occlusion and optic neovascularization. Arch Ophthalmol 1978; 96: 451-2.

10 Brown GC, Magargal LE, Simeone FA, Goldberg RE, Federman JL, Benson WE. Arterial obstruction and ocular neovascularization. Ophthalmology (Rochester) 1982; 89: 139-46. 